\title{
Synthesis and study of the chiral magnetic system $\operatorname{EuIr}_{2} \mathbf{P}_{2}$
}

\author{
D. G. Franco $\circledast^{1,2, *}$ and C. Geibel $\oplus^{1}$ \\ ${ }^{1}$ Max Planck Institute for Chemical Physics of Solids, D-01187 Dresden, Germany \\ ${ }^{2}$ Centro Atómico Bariloche and Instituto Balseiro - Comisión Nacional de Energía Atómica (CNEA), Universidad Nacional de Cuyo, \\ Consejo Nacional de Investigaciones Científicas y Técnicas (CONICET), Avenida E. Bustillo 9500, R8402AGP San Carlos de Bariloche, \\ Río Negro, Argentina
}

(Received 7 December 2020; revised 25 May 2021; accepted 27 July 2021; published 11 August 2021)

\begin{abstract}
Chiral materials, where no improper symmetry operations such as inversion are present, are systems prone to the appearance of a skyrmion lattice. Recently it has been shown theoretically that not only ferromagnets (FMs) but also antiferromagnets (AFMs) can host such kind of phases. In this work we study a new candidate for AFM skyrmions, EuIr $\mathrm{P}_{2}$, by means of magnetization and specific heat measurements on poly and single crystals. X-ray diffraction confirms a trigonal chiral crystal structure, where europium ions form helices along the $c$ direction. In spite of predominantly FM interactions, $\mathrm{Eu}^{2+}$ ions order antiferromagnetically at $T_{N_{1}}=5 \mathrm{~K}$ in what seems to be an incommensurate amplitude-modulated magnetic state where the moments are oriented mainly along the $c$ direction. A second magnetic transition takes place at $T_{N_{2}}=2.9 \mathrm{~K}$, involving the ordering of an in-plane component of the Eu moment likely resulting in an equal-moment structure. Specific heat data show a tail above $T_{N_{1}}$. Accordingly the magnetic entropy at $T_{N_{1}}$ is strongly reduced in comparison to the expected $R \ln 8$ value. This evidences a significant amount of frustration. A simple analysis based on a Heisenberg model indicates that the observed properties imply the presence of several relevant interactions, with competing FM and AFM ones resulting in frustration. Thus $\operatorname{EuIr}_{2} \mathrm{P}_{2}$ is a new interesting magnetic system, where chirality and frustration might result in unconventional magnetic textures.
\end{abstract}

DOI: 10.1103/PhysRevB.104.054416

\section{INTRODUCTION}

Magnetic intermetallic systems based on rare-earth metals are usually described based on two magnetic couplings: the indirect Ruderman-Kittel-Kasuya-Yosida (RKKY) interaction, which tends to order the system, and the Kondo effect which screens the moments at low temperature. The interplay between these two couplings can be represented in the so-called Doniach phase diagram $[1,2]$, where interesting phenomena appear as a function of a nonthermal tuning parameter, such as unconventional superconductivity and non-Fermi-liquid behavior $[3,4]$.

In this context magnetic frustration arises as a new playground for exotic physics. A system is magnetically frustrated when it is not possible to simultaneously minimize all interactions. Such kind of situation can occur either because of a particular geometry or because there are several competing exchange interactions. In both cases long-range order is hindered and novel ground states can emerge, such as a spin liquid [5,6]. Besides, magnetic frustration has been recognized as a relevant factor in the origin of ferroelectricity in multiferroic materials with helical magnetic ordering $[7,8]$.

\footnotetext{
*diego.franco@cab.cnea.gov.ar
}

Published by the American Physical Society under the terms of the Creative Commons Attribution 4.0 International license. Further distribution of this work must maintain attribution to the author(s) and the published article's title, journal citation, and DOI. Open access publication funded by the Max Planck Society.
Chirality can also lead to unusual ground states, such as spin textures known as a skyrmion lattice [9]. The first experimental identification of such kind of phase on a bulk material was reported on metallic $\mathrm{MnSi}[10,11]$, and later $\mathrm{Fe}_{0.8} \mathrm{Co}_{0.2} \mathrm{Si}$ [12] and $\mathrm{Cu}_{2} \mathrm{OSeO}_{3}$ were also identified as skyrmion-hosting bulk compounds $[13,14]$. All of these ferromagnetic systems crystallize in the same crystal structure, space group $P 2{ }_{1} 3$, which is one of the few that lacks not only a center of inversion but also all kinds of improper symmetry operations. Therefore $P 2{ }_{1} 3$ is a chiral space group [15]. The lack of improper symmetry operations allows Dzyaloshinskii-Moriya (DM) interactions [16,17], which have been identified as fundamental to produce skyrmion phases.

It has been predicted theoretically that antiferromagnetic systems are also able to stabilize spin textures $[18,19]$. These results encourage the search for new candidate skyrmion antiferromagnetic materials. The antiferromagnetic compound EuPtSi, which crystallizes in the cubic $P 2{ }_{1} 3$ structure like $\mathrm{MnSi}$, has been recently studied and it has been suggested to present the hallmark of the formation of a skyrmion lattice [20-22].

Among 122 compounds EuIr $\mathrm{P}_{2}$ could present interesting and new physical properties. As an intermetallic compound it could present competing magnetic interactions. Besides, it crystallizes in the trigonal space group $P 3_{2} 21$, a chiral space group like $P 2{ }_{1} 3$, and therefore DM interactions are expected to be present. For a 122 material this trigonal structure is quite special: while many intermetallic compounds crystallize in the well-known tetragonal $\mathrm{ThCr}_{2} \mathrm{Si}_{2}$ or $\mathrm{CaBe}_{2} \mathrm{Ge}_{2}$ crystal structure types, to our knowledge only three compounds crystallize 
in the trigonal $P 3_{2} 21$ crystal structure: $\mathrm{CaIr}_{2} \mathrm{P}_{2}, \operatorname{SrIr}_{2} \mathrm{P}_{2}$, and $\mathrm{EuIr}_{2} \mathrm{P}_{2}[23,24]$. Therefore the europium compound might be the only magnetic 122 system with this particular crystal structure, which is expected to have a strong influence on the magnetic properties. Early work evidenced an $\mathrm{Eu}^{2+}$ state, which bears a large, isotropic magnetic moment. However the magnetic properties were not investigated in detail [23]. We present in this work the synthesis of polycrystalline and single-crystal samples of $\mathrm{EuIr}_{2} \mathrm{P}_{2}$ and the study of the magnetic properties by means of magnetization and specific heat measurements.

\section{EXPERIMENTAL DETAILS}

Polycrystalline $\mathrm{EuIr}_{2} \mathrm{P}_{2}$ samples were prepared heating high-purity Eu (Ames Laboratory), Ir powder (99.99\%; ChemPur), and red P (99.999\%; Alfa-Aesar) pieces at high temperatures in evacuated quartz ampules with aluminum oxide or carbon glass crucibles. Stoichiometric amounts of the elements were mixed and heated several times at progressively higher temperatures, with intermediate regrindings to ensure homogeneity. The best-quality samples were obtained with a final annealing at $1100{ }^{\circ} \mathrm{C}$. Polycrystalline $\operatorname{SrIr}_{2} \mathrm{P}_{2}$ was synthesized as the nonmagnetic counterpart for specific heat measurements, using $\mathrm{Sr}$ (Ames Laboratory). In a first step $\mathrm{SrIr}_{2}$ was obtained heating the elements in a Ta crucible at $950^{\circ} \mathrm{C}$ for several hours and cooling slowly down to $700^{\circ} \mathrm{C}$. To avoid strontium deficiency a small amount of strontium excess was used. In a second step red phosphorus was added. This mixture was heated up to a maximum temperature of $950{ }^{\circ} \mathrm{C}$, with intermediate regrindings as in the europium sample.

Phase purity and crystal structure analysis were performed by $\mathrm{x}$-ray powder diffraction measurements taken at room temperature on a STOE Stadip MP instrument with $\mathrm{Cu} \mathrm{K}_{\alpha 1}$ radiation in transmission mode. Rietveld refinements of the collected data were done with the FullProf program [25] and the crystal structure was drawn using VESTA [26].

$\mathrm{EuIr}_{2} \mathrm{P}_{2}$ single crystals were obtained by arc-melting a polycrystalline sample synthesized as described previously. The typical size of the needle-like single crystals was $\sim 0.70 \times 0.10 \times 0.10 \mathrm{~mm}^{3}$. The composition homogeneity was characterized by microprobe analysis with energydispersive $\mathrm{x}$-ray spectroscopy (EDX). The single crystals were oriented with a Laue machine. We found in all cases that the longest crystal length (the needle axis) corresponds to the crystallographic $c$ direction.

Magnetization data on polycrystalline and single crystals of $\operatorname{EuIr}_{2} \mathrm{P}_{2}$ were taken in a Quantum Design SQUID VSM magnetometer down to $1.8 \mathrm{~K}$ and up to $70 \mathrm{kOe}$. The single crystals were oriented with the applied field $H$ parallel and perpendicular to the $c$ crystallographic direction. Specific heat measurements were performed in a Quantum Design PPMS system with a ${ }^{3} \mathrm{He}$ option on polycrystalline samples.

\section{RESULTS AND DISCUSSION}

Rietveld refinement of the x-ray powder diffraction data indicates a single-crystalline phase for $\operatorname{EuIr}_{2} \mathrm{P}_{2}$ while for $\mathrm{SrIr}_{2} \mathrm{P}_{2}$ a small amount of $\operatorname{IrP}_{2}$ was found (less than $4 \%$ weight). The results confirm for both 122 phases the trigo-
TABLE I. Crystal structure parameters for $\operatorname{EuIr}_{2} \mathrm{P}_{2}$ and $\operatorname{SrIr}_{2} \mathrm{P}_{2}$, space group $P 3_{2} 21$, as obtained from powder Rietveld refinements.

\begin{tabular}{lcccc}
\hline \hline & & $\operatorname{EuIr}_{2} \mathrm{P}_{2}$ & $\operatorname{SrIr}_{2} \mathrm{P}_{2}$ \\
\hline Cell parameter $(\AA)$ & & $\begin{array}{l}a=6.67303(6) \\
c=7.05757(8)\end{array}$ & $a=6.70841(5)$ \\
& & & & \\
Atomic positions: & & & & \\
$\mathrm{Eu} / \mathrm{Sr}$ & $3 b$ & $\left(x, 0, \frac{1}{6}\right)$ & $x=0.5999(3)$ & $x=0.6003(4)$ \\
$\mathrm{Ir}$ & $3 a$ & $\left(x, 0, \frac{2}{3}\right)$ & $x=0.5179(2)$ & $x=0.5161(7)$ \\
$\mathrm{Ir}$ & $3 b$ & $\left(x, 0, \frac{1}{6}\right)$ & $x=0.1299(2)$ & $x=0.1295(1)$ \\
$\mathrm{P}$ & $6 c$ & $(x, y, z)$ & $x=0.2144(9)$ & $x=0.2223(6)$ \\
& & & $y=0.8751(9)$ & $y=0.8791(6)$ \\
& & & $z=0.4506(8)$ & $z=0.4599(5)$ \\
\hline \hline
\end{tabular}

nal crystal structure $P 3_{2} 21$, where there are three formulas per unit cell $(Z=3)$. Table I summarizes the refined cell parameters and atomic positions. The obtained values are in good agreement with a previous report [23]. The crystal structure is schematically shown in Fig. 1, focusing on Eu ions only. From the $a b$ plane view (top) a triangular-like pattern is clearly seen; however the Eu ions are not on the same plane (bottom). Europium ions form helices along the $c$ direction, revealing the symmetry operation $3_{2}$ (a screw axis). An animation of the crystal structure is available in the Supplemental Material [27]. Each europium is shared by two helices and consequently has four nearest neighbors (nn). The two next-nearest-neighbors (nnn) correspond to europium ions on the side helices. Finally, the next-next-nearest neighbors (nnnn) correspond to the second neighbor on the same helix. The distances are $\sim 4.25 \AA, \sim 5.18 \AA$, and $\sim 5.88 \AA$ for $\mathrm{nn}, \mathrm{nnn}$, and nnnn, respectively.

In what follows we will describe first the magnetization and specific heat measurements on the powder samples and later the anisotropic phenomenology observed on single crystals.

A general overview of the magnetization measurements is presented in Fig. 2. For all applied magnetic fields and for temperatures between $200 \mathrm{~K}$ and $300 \mathrm{~K}$ a Curie-Weiss behavior is found: $M / H=c /\left(T+\Theta_{\mathrm{CW}}\right)$, where $c$ is the Curie constant and allows the calculation of the effective magnetic moment $\left(\mu_{\text {eff }}\right)$, and $\Theta_{\mathrm{CW}}$ is the Curie-Weiss temperature. For $H=70 \mathrm{kOe}$ the obtained parameters are $\mu_{\text {eff }}=7.98(5) \mu_{B}$ and $\Theta_{\mathrm{CW}}=+9.22(3) \mathrm{K}(H / M$ vs $T$ and the Curie-Weiss fit can be found in the Supplemental Material [27]). The effective magnetic moment corresponds nicely to the expected value for $\mathrm{Eu}^{2+}$ according to Hund's rules: $L=0$ and $J=S=$ $7 / 2$, which gives $\mu_{\mathrm{eff}}^{\mathrm{Eu}^{2+}}=7.94 \mu_{B}$. The positive sign of $\Theta_{\mathrm{CW}}$ indicates predominantly ferromagnetic interactions between europium ions.

The low-temperature magnetization at 1 kOe shows a maximum followed by a steplike behavior upon decreasing the temperature. Increasing the applied magnetic field to $10 \mathrm{kOe}$ shifts both anomalies to lower temperatures, and for higher fields ( 30 and $70 \mathrm{kOe}$ ) the system is already polarized and no anomalies are observed.

The field dependence of the magnetization at $2 \mathrm{~K}$, inset in Fig. 2, has no hysteresis. At high fields a saturation magnetization $m_{\text {sat }} \approx 6.90 \mu_{B}$ f.u. $^{-1}$ is reached, in good agreement with the expected value $m_{\text {sat }}^{\mathrm{Eu}^{2+}}=7 \mu_{B}$ f.u. $^{-1}$. At intermediate 


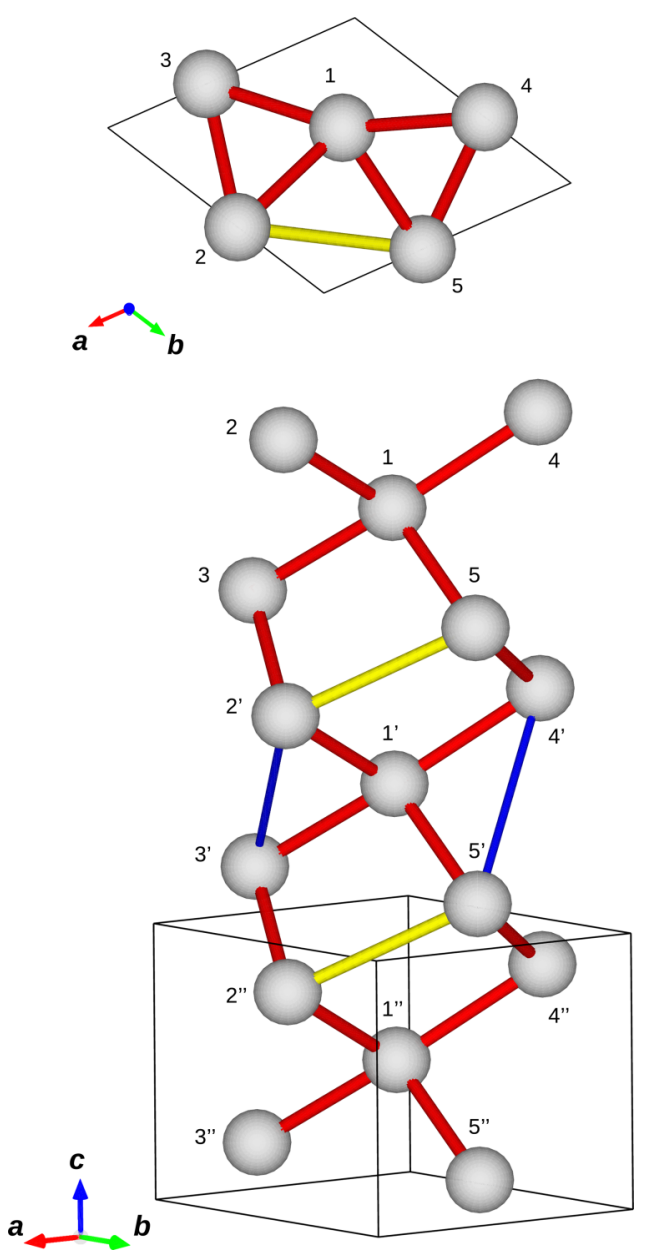

FIG. 1. Crystal structure of $\operatorname{EuIr}_{2} \mathrm{P}_{2}$ showing only europium ions as gray spheres. Red, yellow, and blue lines represent nearest neighbors (nn), next-nearest neighbors (nnn), and next-next-nearest neighbors (nnnn), respectively. Following the red lines one can see the europium helices parallel to the $c$ direction. For simplicity just some representative nnn and nnnn interactions are shown. Atoms with the same number label on the lower figure lie on top of each other on the upper figure. The unit cell is indicated as black lines.

fields, approximately $15 \mathrm{kOe}$ at $2 \mathrm{~K}$, a signature similar to a metamagnetic-like transition is found.

The lack of remanent magnetization in the field dependence of the magnetization, together with the maximum observed in the magnetization versus temperature data, indicates that $\operatorname{EuIr}_{2} \mathrm{P}_{2}$ orders antiferromagnetically at low temperatures, followed by a second transition to a different AFM state. However a positive $\Theta_{\mathrm{CW}}$ is obtained from the high-temperature magnetization data, suggesting a competition between antiferromagnetic and ferromagnetic interactions. We will come back to this issue later when discussing the exchange interactions and the magnetic entropy. The ordering temperatures obtained from $\partial M / \partial T$ vs $T$ at $1 \mathrm{kOe}$ are $T_{N_{1}}=5.02(5) \mathrm{K}$ and $T_{N_{2}}=2.94(5) \mathrm{K}$.

Possible magnetism coming from iridium ions can safely be disregarded as the experimental parameters which give direct information about the nature of the magnetic ions, $\mu_{\text {eff }}$

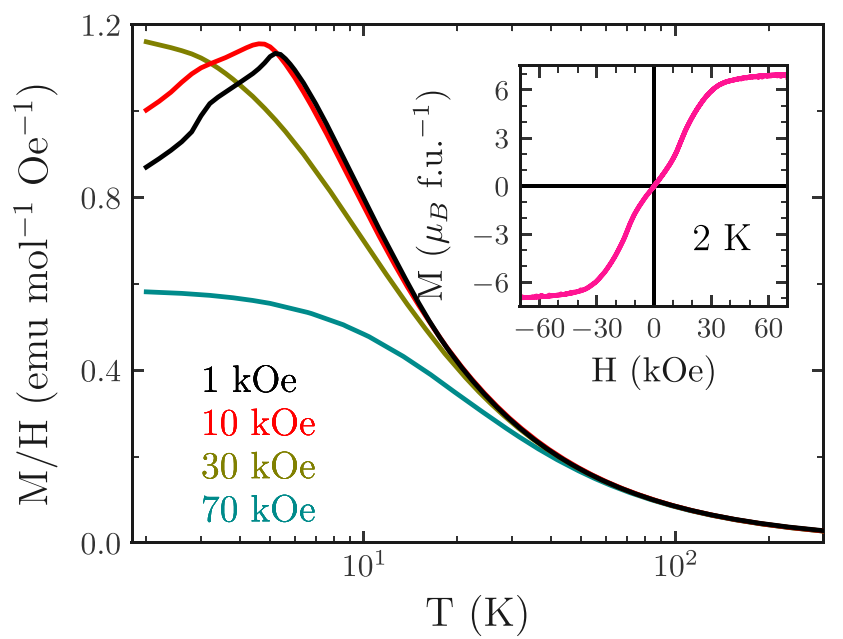

FIG. 2. $M / H$ temperature dependence for $\operatorname{EuIr}_{2} \mathrm{P}_{2}$ powder sample taken at different applied magnetic fields as indicated. Note the logarithmic horizontal axis. The inset shows the isothermal field dependence of the magnetization at $2 \mathrm{~K}$.

and $m_{\text {sat }}$, are in very good agreement with the $\mathrm{Eu}^{2+}$-only values.

Figure 3(a) shows the specific heat data as a function of temperature on a polycrystalline pellet. As in the susceptibility data two clear anomalies are observed, confirming the bulk nature of the magnetic transitions on $\operatorname{EuIr}_{2} \mathrm{P}_{2}$. An applied magnetic field shifts $T_{N_{1}}$ and $T_{N_{2}}$ to lower temperatures, in accordance with $M(T)$ results. At $50 \mathrm{kOe}$ the specific heat exhibits just a Schottky-like anomaly, as expected for a fully polarized state of the europium ions.

Considering $C=C_{m}+C_{\mathrm{el}}+C_{\mathrm{ph}}$, where $C_{m}, C_{\mathrm{el}}$, and $C_{\mathrm{ph}}$ stand for the magnetic, electron, and phonon contributions, respectively, in order to analyze $C_{m}$ only we must find a way to evaluate the specific heat coming from the lattice $\left(C_{\mathrm{el}}\right.$ and $C_{\mathrm{ph}}$ ). Next we will describe two different methods to accomplish this subtraction.

At low temperature the electronic specific heat for a metal is given by $C_{\mathrm{el}}=\gamma T$, while a Debye model gives $C_{\mathrm{ph}}=\beta T^{3}$ for the phonon contribution. The fit of the measured data for $\operatorname{EuIr}_{2} \mathrm{P}_{2}$ at zero field between 17 and $32 \mathrm{~K}$, where no magnetic signal is expected, can provide a reliable $\beta$ value and a rough estimation of $\gamma$. We get a Sommerfeld coefficient $\gamma=$ 23(5) $\mathrm{mJ} \mathrm{mol}^{-1} \mathrm{~K}^{-2}$ and $\beta=4.39(8) \times 10^{-4} \mathrm{~J} \mathrm{~mol}^{-1} \mathrm{~K}^{-4}$. The $C T^{-1}$ vs $T^{2}$ plot and the corresponding fit can be found in the Supplemental Material [27]. With these parameters we can calculate $C_{\mathrm{el}}$ and $C_{\mathrm{ph}}$ for all temperatures and subtract this from the total $C$ in order to get $C_{m}$ (method 1). The large uncertainty in the $\gamma$ coefficient turns out to be irrelevant for the present analysis. As can easily be seen in Fig. 3(b), the magnetic contribution $C_{m}(T) / T$ is of the order of $2 \mathrm{~J} \mathrm{~mol}^{-1} \mathrm{~K}^{-2}$, thus 2 orders of magnitude larger than the estimated electronic part. Therefore the electronic part can almost be neglected. From $\beta$ the Debye temperature is found to be $\Theta_{D}\left(\operatorname{EuIr}_{2} \mathrm{P}_{2}\right)=281 \mathrm{~K}$.

The second method (method 2) is to deduce the lattice contribution from the specific heat of a nonmagnetic homologue compound. In this case we measured the isostructural material $\mathrm{SrIr}_{2} \mathrm{P}_{2}$; see Fig. 3(a). Before subtracting the phonon 


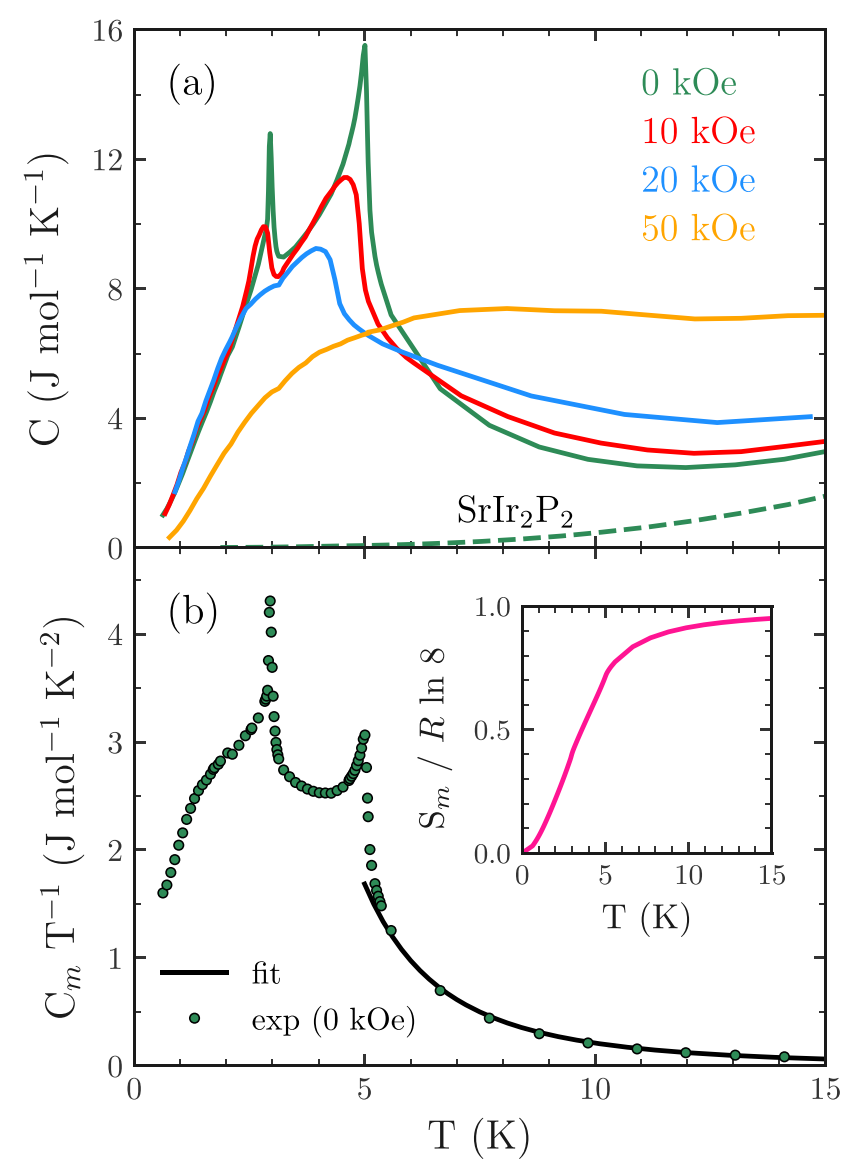

FIG. 3. (a) Specific heat versus temperature for a polycrystalline $\mathrm{EuIr}_{2} \mathrm{P}_{2}$ sample at different applied magnetic fields as indicated. The data for the nonmagnetic reference compound $\operatorname{SrIr}_{2} \mathrm{P}_{2}$ at zero field is also shown for comparison. (b) $C_{m} T^{-1}$ vs $T$ at zero applied magnetic field. Dots: Experimental $\operatorname{EuIr}_{2} \mathrm{P}_{2}$, black line: high-temperature fit following $C_{m}(T) \propto T^{-2}$ law. For details see text. Inset: Magnetic entropy normalized to the expected value for an $S=7 / 2$ system.

contribution from the EuIr $\mathrm{P}_{2}$ data, $C_{\mathrm{ph}}$ of $\operatorname{SrIr}_{2} \mathrm{P}_{2}$ was scaled using the mass ratio [28].

The two described approaches resulted in very similar $C_{m}(T)$. A comparison between the results obtained by methods 1 and 2 can be found in the Supplemental Material [27]. A reliable lattice subtraction is very important, as it directly affects the $C_{m}$ values and, consequently, the magnetic entropy (see below). The experimental $C_{m}$ at zero field is plotted in Fig. 3(b). In the following paragraphs we will analyze and discuss different features in the specific heat curve: the jump at $T_{N_{1}}$, then the low-temperature behavior, and finally the hightemperature region.

Within a mean-field approximation the expected height of the peak at the ordering temperature for a $7 / 2$-spin equalmoment (EM) system, where all magnetic moments are the same at all sites, is $\Delta C_{m}^{\mathrm{EM}}=20.15 \mathrm{~J} \mathrm{~mol}^{-1} \mathrm{~K}^{-1}$. This category includes ferromagnets and simple antiferromagnets, but also helical and cycloidal magnetic structures. It is important to notice that while the first two magnetic structures are commensurate with the lattice periodicity, the later two are usually incommensurate. The specific heat jump for $\operatorname{EuIr}_{2} \mathrm{P}_{2}$ is reduced compared with the EM mean-field expectation, which suggests a different kind of magnetic behavior. Another possible scenario is an incommensurate amplitude-modulated (AM) magnetic structure, where the magnetic moment amplitude varies periodically from one site to another. According to a study on Gd systems $\Delta C_{m}^{\mathrm{AM}}=(2 / 3) \Delta C_{m}^{\mathrm{EM}}=$ $13.43 \mathrm{~J} \mathrm{~mol}^{-1} \mathrm{~K}^{-1}[29,30]$. As $\mathrm{Eu}^{2+}$ and $\mathrm{Gd}^{3+}$ are both $7 / 2-$ spin systems, they are magnetically equivalent. Besides, AM magnetic phases are usually expected to transform to EM structures at lower temperatures, as for example in $\mathrm{EuAs}_{3}$ and EuPbSb [31,32]. The specific heat jump in $\operatorname{EuIr}_{2} \mathrm{P}_{2}$ at $T_{N_{1}}$ seems to be closer to the expected value for an AM structure, which suggests a transition to an incommensurate state at $T_{N_{1}}$. In such a case $T_{N_{2}}$ could be a transition to an EM structure. AM structures are normally explained in rare-earth metals and alloys as a consequence of the oscillatory RKKY exchange interaction. This coupling gives rise in fact to a wide variety of magnetic orderings [33]. However $\mathrm{EuIr}_{2} \mathrm{P}_{2}$ is a noncentrosymmetric system, where DM interactions could also be relevant. It has been reported that such antisymmetric exchange can be responsible for the formation of several incommensurate magnetic structures $[34,35]$. Thus both kinds of exchange interactions can be playing an important role in $\operatorname{EuIr}_{2} \mathrm{P}_{2}$.

Now we turn to the low-temperature specific heat. Below the ordering temperature the $T$ dependence of $C_{m}(T)$ in a $S=$ $7 / 2$ system is very different from that in a system with much smaller $S$ value, e.g., $S=1 / 2$. The large degeneracy of an $S=7 / 2$ state results in many magnon branches, corresponding to the eight equally spaced singlets of a polarized $S=7 / 2$ single ion, with the excitation energy of the first branch being much smaller (in relation to $T_{N}$ ) than in an $S=1 / 2$ system, in correspondence with the much smaller energy of the first excited singlet in the single-ion case. This gives rise to a Schottky-like anomaly around $T / T_{N} \approx 0.3[30,36]$, somehow hidden due to the peak at $T_{N_{2}}$ in $\operatorname{EuIr}_{2} \mathrm{P}_{2}$ but still noticeable; see Fig. 3(b).

Finally, above $T_{N_{1}}$ a tail is observed in $C_{m}(T)$, which could be related to frustration or low dimensionality. Notably, this tail can be nicely fitted with $C_{m}=d_{2} / T^{2}$, which is expected for a local moment system with a finite range of interactions. We obtain $d_{2}=211 \mathrm{~J} \mathrm{~K} \mathrm{~mol}^{-1}$. This allows us to gain more insight into this system using simple, wellestablished approaches based on the Heisenberg model $H=$ $\sum_{i j} J_{i j} S_{i} \cdot S_{j}$. Here $J_{i j}$ is the exchange interaction in units of temperature between spins $S_{i}$ and $S_{j}$, with $J_{i j}<0$ indicating a ferromagnetic and $J_{i j}>0$ an antiferromagnetic coupling. The high-temperature expansion (HTE) is a simple and universal approach where measurable properties are written as Taylor series in ascending powers of the reciprocal temperature, allowing the determination of $J_{i j}$ from experimental data $[37,38]$. In particular, the susceptibility and the magnetic specific heat can be written as $\chi(\beta)=\sum_{n=1}^{\infty} c_{n} \beta^{n}$ and $C_{m}(\beta)=\sum_{n=2}^{\infty} d_{n} \beta^{n}$, respectively, where $\beta=\left(k_{B} T\right)^{-1}$, and the coefficients $c_{n}$ and $d_{n}$ depend on the details of the magnetic system under consideration. The expansions of the susceptibility and specific heat show that

$$
\begin{aligned}
\Theta_{\mathrm{CW}} & =\frac{c_{2}}{c_{1}}=-\frac{1}{3} S(S+1) \sum_{i} J_{i}, \\
d_{2} & =\frac{1}{6} R[S(S+1)]^{2} \sum_{i} J_{i}^{2},
\end{aligned}
$$


where $\Theta_{\mathrm{CW}}$ is the Curie-Weiss temperature, $c_{1}, c_{2}$, and $d_{2}$ are expansion coefficients for the susceptibility and the specific heat, $S$ is the spin quantum number, $R$ is the gas constant, the interactions $J_{i}$ are given in $\mathrm{K}$, and the sums are over the different neighbors of a magnetic ion. These equations allow the calculation of $\sum J_{i}$ and $\sum J_{i}^{2}$ from experimental $\Theta_{\mathrm{CW}}$ and $d_{2}$.

A simple $J_{1}-J_{2}$ model, that is just nn and nnn interactions (see Fig. 1), has two solutions: $J_{1}=-0.201 \mathrm{~K}$ and $J_{2}=-0.475 \mathrm{~K}$, and $J_{1}=-0.384 \mathrm{~K}$ and $J_{2}=-0.110 \mathrm{~K}$. In both cases $J_{1}$ and $J_{2}$ are ferromagnetic in nature, which does not explain the antiferromagnetic state found. This apparent contradiction is obviously a consequence of an oversimplified model with only two interactions. If we take into account also nnnn interactions $J_{3}$, we obtain a range of parameters which can be classified into two groups: ferromagnetic cases, again not of interest in the present material, and solutions where there is just one antiferromagnetic interaction, either $J_{1}, J_{2}$, or $J_{3}$. Just as an example, the parameters with the strongest antiferromagnetic $J_{2}$ are $J_{1}=J_{3}=-0.263 \mathrm{~K}$ and $J_{2}=0.174 \mathrm{~K}$. Interestingly in any of those cases, where there is one $J_{i}>0$, magnetic frustration arises as a consequence of competing AFM and FM exchange path. Such scenario would explain the observed properties in $\operatorname{EuIr}_{2} \mathrm{P}_{2}$, a system with positive Curie-Weiss temperature but nevertheless an antiferromagnetic macroscopic order, caused by frustration. Going beyond this level of analysis requires additional information, such as the knowledge of the magnetic structures. Therefore this is a topic for future studies.

The magnetic entropy $S_{m}(T)$, obtained integrating $C_{m} T^{-1}$ vs $T$ at zero field, is shown in Fig. 3(b) [39]. The expected saturation value $S_{m}(\infty)$ for a magnetic ion is $R \ln (2 J+1)$, which for spin $J=7 / 2$ gives $R \ln 8$. In magnetic systems without frustration and thus without enhanced fluctuations, one expects $S_{m}\left(T_{N}\right) \approx 0.9 S_{m}(\infty)$. In $\operatorname{EuIr}_{2} \mathrm{P}_{2}, S_{m}\left(T_{N_{1}}\right)$ is much smaller, only about $72 \%$ of $S_{m}(\infty)$. The remaining entropy is collected in the large tail in $C_{m}(T)$ above $T_{N_{1}}$, which extend to at least 3 times $T_{N_{1}}$. As stated before for the large tail in $C_{m}(T)$ for $T>T_{N_{1}}$, also the comparatively small value of $S_{m}\left(T_{N_{1}}\right)$ is an indication of stronger fluctuations. In the present case the most likely origin for these stronger fluctuations is frustration, as also suggested by our simple analysis above.

Now we focus on $\operatorname{EuIr}_{2} \mathrm{P}_{2}$ single-crystal properties. Two different field configurations were used: $H \| c$ and $H \perp c$. The high-temperature magnetization data on both directions are well described by a Curie-Weiss law (shown in the Supplemental Material [27]). The obtained $\mu_{\text {eff }}$ are similar to the powder value, while $\Theta_{\mathrm{CW}}$ are slightly smaller, but still positive. Figure 4 draws attention to the low-temperature magnetization for some selected and representative applied magnetic fields. Although $\mathrm{Eu}^{2+}$ has no single-ion anisotropy (as $L=0$ ), significant anisotropic behavior is found, especially below $T_{N_{1}}$. When a $0.5 \mathrm{kOe}$ field is applied parallel to the $c$ direction a maximum in the magnetization is observed at $T_{N_{1}}$, whereas $T_{N_{2}}$ is completely absent. For $H \perp c$ a maximum value is reached at $T_{N_{1}}$; then the magnetization remains practically constant and finally there is a steplike anomaly at $T_{N_{2}}$. The increase of the applied magnetic fields shifts all these features to lower temperatures, turning the steplike behavior at
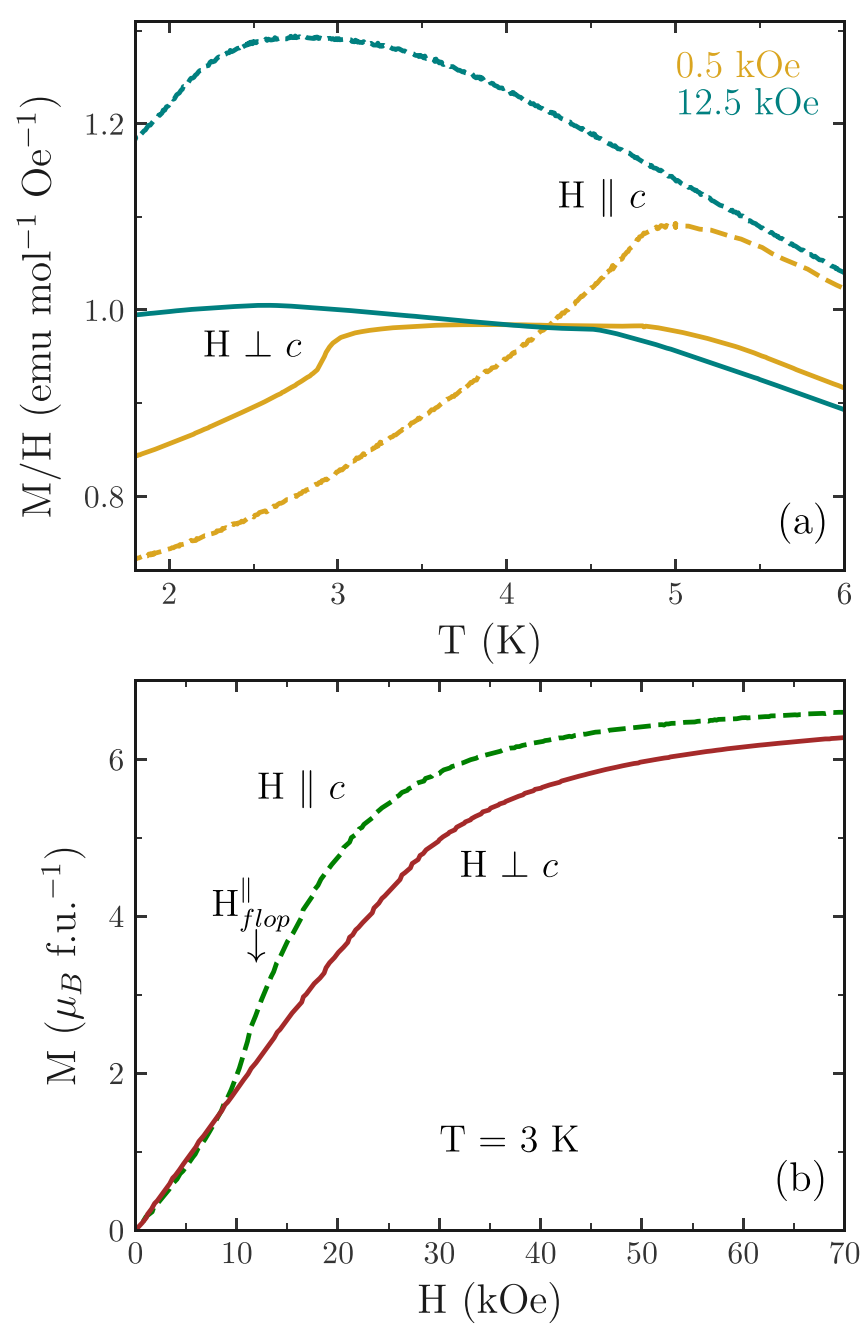

FIG. 4. $M / H$ versus temperature for selected applied magnetic fields (a) and field dependence of the magnetization at $3 \mathrm{~K}$ (b) for EuIr $_{2} \mathrm{P}_{2}$ single crystals. The crystal and field configurations are $H \| c$ (dashed lines) and $H \perp c$ (continuous lines) for both panels.

$T_{N_{2}}$ to a kink, still noticeable at $12.5 \mathrm{kOe}$, until eventually it disappears.

The anisotropy found in $\operatorname{EuIr}_{2} \mathrm{P}_{2}$ could be surprising because $\mathrm{Eu}^{2+}$ has no single-ion anisotropy, which is usually the largest contribution to magnetic anisotropy in rare-earth compounds. However there are other sources of anisotropy such as dipolar and exchange anisotropy. For several centrosymmetric ferromagnets and antiferromagnets based on $\mathrm{Gd}^{3+}$ (also an $L=0$ ion) the dipolar interaction has been identified as the dominating source of anisotropy by means of an analytical method using the propagation vector of the magnetic structure and the magnetic moment orientation [40,41]. From our $\mathrm{EuIr}_{2} \mathrm{P}_{2}$ single-crystal measurements (see following paragraphs) we have evidence regarding the magnetic moment orientation, but the propagation vector is unknown. Therefore it is not possible to apply this numerical method to $\operatorname{EuIr}_{2} \mathrm{P}_{2}$ and the origin of the magnetic anisotropy remains for further studies. Nevertheless it is important to stress that the validity of this analytical method in noncentrosymmetric materials is 


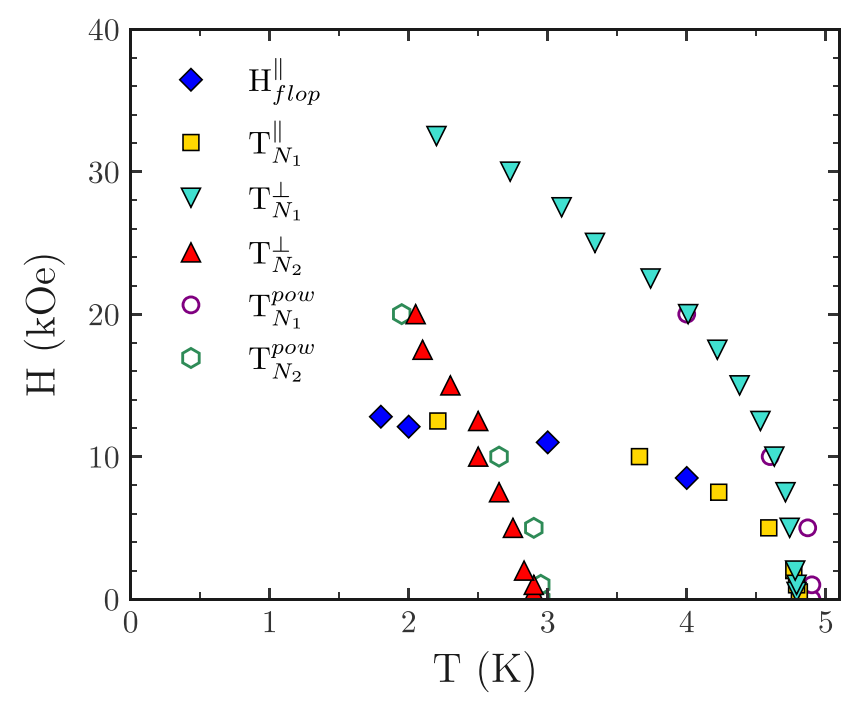

FIG. 5. Magnetic phase diagram $H-T$ for $\operatorname{EuIr}_{2} \mathrm{P}_{2}$. Filled symbols are the transitions obtained on single crystals. Triangles and squares correspond to transitions for $H \perp c$ and $H \| c$, respectively, as indicated. $H_{\text {flop }}^{\|}$corresponds to the spin-flop rearrangement in $M$ vs $H$ curves. Empty symbols are transition temperatures obtained from specific heat measurements on the powder sample.

unclear, as it does not take into account DM interactions, which might be relevant in systems such as $\operatorname{EuIr}_{2} \mathrm{P}_{2}$.

The isothermal field dependence of the magnetization for EuIr $_{2} \mathrm{P}_{2}$ single crystals is shown in Fig. 4(b). A linear dependence is seen up to $\sim 25 \mathrm{kOe}$ for $H \perp c$, followed by a slower increase up to the highest attainable field, with no complete saturation. For $H \| c$ the low-field magnetization evolves with the same slope as in the other field direction but a stronger increase on the magnetization is found at $\sim 10 \mathrm{kOe}$, resembling a metamagnetic transition.

For a simple antiferromagnet with two sublattices, below $T_{N}$ the susceptibility goes to zero on cooling when the field is applied parallel to the direction of the AFM-ordered moments $\left(\chi^{\|}\right)$, while for the field applied perpendicular to it $\left(\chi^{\perp}\right)$ the signal remains constant [42]. From the phenomenology observed around $T_{N_{1}}$ in $M(T)$ and $M(H)$ curves, both in Fig. 4, one can deduce that for $\mathrm{EuIr}_{2} \mathrm{P}_{2}$ in zero field the AFM-ordered moments point mainly along the crystallographic $c$ direction, that is, along the direction of the europium helices. Regarding the field dependence, the applied magnetic field produces at $H_{\text {flop }}^{\|}$a spin-flop, that is, a reorientation perpendicular to the field of the spins. At higher fields the magnetization increases gradually, with no clear critical field coming from the full orientation of the spins along the magnetic field (spin-flip). Accordingly the $a b$ plane is a magnetic hard plane. However, the step in $(M / H)^{\perp}$ at $T_{N_{2}}$ at $0.5 \mathrm{kOe}$ indicates that $T_{N_{2}}$ is very likely associated with the ordering of an in-plane component of the Eu moment.
Figure 5 shows the $H$-T phase diagram for $\operatorname{EuIr}_{2} \mathrm{P}_{2}$. For the field along the $c$ direction $(H \| c), T_{N_{1}}^{\|}$obtained from magnetization data coincides with the spin-flop reorientation obtained from the field dependence of the magnetization, $H_{\text {flop }}^{\|}$. It is observed that this magnetic transition is rapidly suppressed by the field. On the other hand, for the field in the $a b$ plane, both transitions survive up to much higher magnetic fields, evidencing a clear anisotropy in the AFM regime in this compound. This behavior in the observed magnetic properties does not imply a large anisotropy in the interactions. Even the tiniest anisotropy in the interaction (or at the local moment) shall result that in the ordered state the moment prefers to order along one particular direction. The magnitude of the anisotropy in the interactions is then better reflected, e.g., in the size of the magnetic field required to induce a spin-flop. In the present case, taking into account the huge $\mathrm{Eu}^{2+}$ moment $\left(7 \mu_{B}\right)$, this field is quite small, about $10 \mathrm{kOe}$. Possible origins for such anisotropies in Eu systems are, e.g., the dipolar interaction discussed before, which is comparatively large because of the large moment, or the anisotropy in the exchange, which could be induced by the large spin-orbit coupling at the Ir ligands.

\section{CONCLUSIONS}

We synthesized polycrystalline samples and single crystals of the chiral compound $\operatorname{EuIr}_{2} \mathrm{P}_{2}$, and studied in detail the magnetic properties of this peculiar system. The magnetic properties at high temperature are in excellent agreement with a fully localized $\mathrm{Eu}^{2+}$ state. Susceptibility and specific heat data both indicate a first magnetic transition to an AFM state at $T_{N_{1}}=5 \mathrm{~K}$ followed by a second transition at $T_{N_{2}}=2.9 \mathrm{~K}$ from an AFM1 to an AFM2 state. The temperature dependence of the specific heat suggests the AFM1 state to have an incommensurate amplitude modulated structure and the AFM2 state to be an equal moment state. An analysis of the specific heat and the entropy indicate the presence of frustration, which is likely due to competing FM and AFM interactions. Magnetization data on single crystals reveal a clear anisotropy in the ordered state, which indicates the moment to order predominantly along the $c$ direction, but with an in-plane component ordering at $T_{\mathrm{N}_{2}}$. The observation of a complex magnetic phase diagram in this compound with a chiral structure makes it a good candidate for the appearance of unusual, skyrmion-like structures. Thus our results prompt an in depth study of this compound to determine the field and temperature dependent magnetic structures.

\section{ACKNOWLEDGMENTS}

D.G.F. would like to thank R. Cardoso-Gil and J. AndradeGamboa for fruitful discussions regarding chirality and crystallography.
[1] S. Doniach, The Kondo lattice and weak antiferromagnetism, Physica B+C 91, 231 (1977).
[2] M. Lavagna, C. Lacroix, and M. Cyrot, Volume collapse in the Kondo lattice, Phys. Lett. A 90, 210 (1982). 
[3] P. Gegenwart, Q. Si, and F. Steglich, Quantum criticality in heavy-fermion metals, Nat. Phys. 4, 186 (2008).

[4] N. D. Mathur, F. M. Grosche, S. R. Julian, I. R. Walker, D. M. Freye, R. K. W. Haselwimmer, and G. G. Lonzarich, Magnetically mediated superconductivity in heavy fermion compounds, Nature (London) 394, 39 (1998).

[5] P. A. Lee, An end to the drought of quantum spin liquids, Science 321, 1306 (2008).

[6] L. Balents, Spin liquids in frustrated magnets, Nature (London) 464, 199 (2010).

[7] S.-W. Cheong and M. Mostovoy, Multiferroics: A magnetic twist for ferroelectricity, Nat. Mater. 6, 13 (2007).

[8] M. V. Gvozdikova, T. Ziman, and M. E. Zhitomirsky, Helicity, anisotropies, and their competition in a multiferroic magnet: Insight from the phase diagram, Phys. Rev. B 94, 020406(R) (2016).

[9] A. N. Bogdanov and U. K. Rößler, Chiral Symmetry Breaking in Magnetic Thin Films and Multilayers, Phys. Rev. Lett. 87, 037203 (2001).

[10] S. Mühlbauer, B. Binz, F. Jonietz, C. Pfleiderer, A. Rosch, A. Neubauer, R. Georgii, and P. Böni, Skyrmion lattice in a chiral magnet, Science 323, 915 (2009).

[11] C. Fleiderer, T. Adams, A. Bauer, W. Biberacher, B. Binz, F. Birkelbach, P. Böni, C. Franz, R. Georgii, M. Janoschek, F. Jonietz, T. Keller, R. Ritz, S. Mühlbauer, W. Münzer, A. Neubauer, B. Pedersen, and A. Rosch, Skyrmion lattices in metallic and semiconducting B20 transition metal compounds, J. Phys.: Condens. Matter 22, 164207 (2010).

[12] W. Münzer, A. Neubauer, T. Adams, S. Mühlbauer, C. Franz, F. Jonietz, R. Georgii, P. Böni, B. Pedersen, M. Schmidt, A. Rosch, and C. Pfleiderer, Skyrmion lattice in the doped semiconductor $\mathrm{Fe}_{1-x} \mathrm{Co}_{x} \mathrm{Si}$, Phys. Rev. B 81, 041203(R) (2010).

[13] T. Adams, A. Chacon, M. Wagner, A. Bauer, G. Brandl, B. Pedersen, H. Berger, P. Lemmens, and C. Pfleiderer, Long-Wavelength Helimagnetic Order and Skyrmion Lattice Phase in $\mathrm{Cu}_{2} \mathrm{OSeO}_{3}$, Phys. Rev. Lett. 108, 237204 (2012).

[14] S. Seki, X. Z. Yu, S. Ishiwata, and Y. Tokura, Observation of skyrmions in a multiferroic material, Science 336, 198 (2012).

[15] G. S. Girolami, X-Ray Crystallography (University Science Books, Mill Valley, California, 2016), Chap. 9.

[16] I. A. Dzyaloshinskii, A thermodynamic theory of "weak" ferromagnetism of antiferromagnetics, J. Phys. Chem. Solids 4, 241 (1958).

[17] T. Moriya, Anisotropic superexchange interaction and weak ferromagnetism, Phys. Rev. 120, 91 (1960).

[18] H. D. Rosales, D. C. Cabra, and P. Pujol, Three-sublattice skyrmion crystal in the antiferromagnetic triangular lattice, Phys. Rev. B 92, 214439 (2015).

[19] R. Keesman, M. Raaijmakers, A. E. Baerends, G. T. Barkema, and R. A. Duine, Skyrmion in square-lattice antiferromagnets, Phys. Rev. B 94, 054402 (2016).

[20] D. G. Franco, Y. Prots, C. Geibel, and S. Seiro, Fluctuationinduced first-order transition in Eu-based trillium lattices, Phys. Rev. B 96, 014401 (2017).

[21] M. Kakihana, D. Aoki, A. Nakamura, F. Honda, M. Nakashima, Y. Amako, S. Nakamura, T. Sakakibara, M. Hedo, T. Nakama, and Y. Onuki, Giant Hall resistivity and magnetoresistance in cubic chiral antiferromagnet EuPtSi, J. Phys. Soc. Jpn. 87, 023701 (2018)

[22] K. Kaneko, M. D. Frontzek, M. Matsuda, A. Nakao, K. Munakata, M. Ohhara, T. Kakihana, Y. Haga, M. Hedo, T. Nakama, and Y. Onuki, Unique helical magnetic order and field-induced phase in trillium lattice antiferromagnet EuPtSi, J. Phys. Soc. Jpn. 88, 013702 (2019).

[23] C. Lux, A. Mewis, S. Junk, A. Gruetz, and G. Michels, Kristallstrukturen und Eigenschaften neuer ternärer Iridiumphosphide, J. Alloys Compd. 200, 135 (1993).

[24] A. Löhken, C. Lux, D. Johrendt, and A. Mewis, Crystal and electronic structures of $\mathrm{AIr}_{2} \mathrm{P}_{2}(\mathrm{~A}: \mathrm{Ca}-\mathrm{Ba}), \mathrm{Z}$. Anorg. Allg. Chem. 628, 1472 (2002).

[25] J. Rodríguez-Carvajal, Recent advances in magnetic structure determination by neutron powder diffraction, Physica B 192, 55 (1993).

[26] K. Momma and F. Izumi, VESTA 3 for three-dimensional visualization of crystal, volumetric, and morphology data, J. Appl. Crystallogr. 44, 1272 (2011).

[27] See Supplemental Material at http://link.aps.org/supplemental/ 10.1103/PhysRevB.104.054416 for the following: an animation of the crystal structure for $\operatorname{EuIr}_{2} \mathrm{P}_{2} ; H / M$ vs $T$ and Curie-Weiss fit at $70 \mathrm{kOe}$ for the powder sample and single crystals with $H \|$ $c$ and $H \perp c ; C T^{-1}$ vs $T^{2}$ at zero applied field and the $\gamma+\beta T^{2}$ fit; $C_{m}$ vs $T$ at zero applied field obtained by methods 1 and 2 .

[28] The renormalization scheme to scale the phonon specific heat of $\operatorname{SrIr}_{2} \mathrm{P}_{2}$ was $\Theta_{D}\left(\operatorname{EuIr}_{2} \mathrm{P}_{2}\right) / \Theta_{D}\left(\operatorname{SrIr}_{2} \mathrm{P}_{2}\right)=\left[\left(m_{\mathrm{Sr}}^{3 / 2}+2 m_{\mathrm{Ir}}^{3 / 2}+\right.\right.$ $\left.\left.2 m_{\mathrm{P}}^{3 / 2}\right) /\left(m_{\mathrm{Eu}}^{3 / 2}+2 m_{\mathrm{Ir}}^{3 / 2}+2 m_{\mathrm{P}}^{3 / 2}\right)\right]^{1 / 3}$, where $m_{i}$ is the atomic mass of the $i$ element.

[29] M. Bouvier, P. Lethuillier, and D. Schmitt, Specific heat in some gadolinium compounds. I. Experimental, Phys. Rev. B 43, 13137 (1991).

[30] J. A. Blanco, D. Gignoux, and D. Schmitt, Specific heat in some gadolinium compounds. II. Theoretical model, Phys. Rev. B 43, 13145 (1991).

[31] T. Chattopadhyay, P. J. Brown, P. Thalmeier, and H. G. v, Schnering, Incommensurate Magnetic Phase in $\mathrm{EuAs}_{3}$ with Zone-Boundary Lock-in, Phys. Rev. Lett. 57, 372 (1986).

[32] P. Bonville, J. A. Hodges, M. Shirakawa, M. Kasaya, and D. Schmitt, Incommensurate modulated magnetic structure in orthorhombic EuPdSb, Eur. Phys. J. B 21, 349 (2001).

[33] R. J. Elliott, editor, Magnetic Properties of Rare Earth Metals (Springer, 1972), Chap. 3.

[34] A. N. Bogdanov, U. K. Rößler, M. Wolf, and K. H. Müller, Magnetic structures and reorientation transitions in noncentrosymmetric uniaxial antiferromagnets, Phys. Rev. B 66, 214410 (2002).

[35] A. Zheludev, G. Shirane, Y. Sasago, N. Koide, and K. Uchinokura, Spiral phase and spin waves in the quasi-twodimensional antiferromagnet $\mathrm{Ba}_{2} \mathrm{CuGe}_{2} \mathrm{O}_{7}$, Phys. Rev. B 54, 15163 (1996).

[36] J. I. Facio, D. Betancourth, P. Pedrazzini, V. F. Correa, V. Vildosola, D. J. García, and P. S. Cornaglia, Why the Co-based 115 compounds are different: The case study of ${\operatorname{Gd} M \mathrm{In}_{5}}_{5}(M=$ Co, Rh, Ir), Phys. Rev. B 91, 014409 (2015).

[37] H.-J. Schmidt, A. Lohmann, and J. Richter, Eighth-order hightemperature expansion for general Heisenberg Hamiltonians, Phys. Rev. B 84, 104443 (2011). 
[38] H.-J. Schmidt, J. Schnack, and M. Luban, Heisenberg exchange parameters of molecular magnets from the high-temperature susceptibility expansion, Phys. Rev. B 64, 224415 (2001).

[39] For computing the entropy below the lowest temperature reached experimentally, $C_{m}(T) / T$ versus $T$ was interpolated linearly to zero at $T=0 \mathrm{~K}$.

[40] M. Rotter, M. Loewenhaupt, M. Doerr, A. Lindbaum, H. Sassik, K. Ziebeck, and B. Beuneu, Dipole interaction and magnetic anisotropy in gadolinium compounds, Phys. Rev. B 68, 144418 (2003).

[41] M. Rotter, M. Loewenhaupt, M. Doerr, A. Lindbaum, and H. Michor, Noncollinear amplitude-modulated magnetic order in Gd compounds, Phys. Rev. B 64, 014402 (2001).

[42] N. A. Spalding, Magnetic Materials, 2nd ed. (Cambridge University Press, University of California, Santa Barbara, 2010), Chap. 8. 